\title{
Relationship between atmospheric aerosol mineral surface area and iron solubility
}

\author{
E. D. INGALL ${ }^{1 *}$, M.F.M. MCDANIEL ${ }^{1}$, P.L. MORTON ${ }^{2}$, \\ E. CASTORINA ${ }^{1}$, R.J. WEBER ${ }^{1}$, R.U. SHELLEY ${ }^{3}$, W.M. \\ LANDING $^{3}$, A.F.LONGO ${ }^{1}$, Y.FENG ${ }^{4}$, B. LAI ${ }^{4}$ \\ ${ }^{1}$ School of Earth and Atmospheric Sciences, Georgia Institute \\ of Technology, Atlanta, GA 30332, USA \\ (*correspondence: ingall@eas.gatech.edu) \\ ${ }^{2}$ National High Magnetic Field Laboratory, Florida State \\ University, Tallahassee, FL 32310, USA \\ ${ }^{3}$ Earth, Ocean and Atmospheric Science, Florida State \\ University, Tallahassee, FL 32306, USA \\ ${ }^{4}$ Argonne National Laboratory, Argonne, IL 60439, USA
}

In approximately $30 \%$ of the ocean, atmospheric aerosol deposition supplies the majority of the key micronutrient, iron, which controls the growth of photosynthetic microorganisms. The factors that control the solubility of aerosol iron, and therefore bioavailability of iron to microorganisms, remain unclear. To better understand controls on aerosol iron solubility, size-fractionated dust aerosols collected shipboard in the Atlantic Ocean were investigated. Samples originated from source regions including the Sahara, North America and the local marine environment. Iron mineralogy, oxidation state, and solubility were examined using wet chemistry and synchrotron-based iron K-edge X-ray absorption near edge structure (XANES) spectroscopy. Soluble iron concentration showed a strong positive correlation with calculated aerosol surface area $\left(\mathrm{R}^{2}=\right.$ 0.81). The iron XANES spectra, for all size ranges, were nearly identical for Saharan samples. Spectra from North American and local atmosphere (marine) provenances exhibited more variation between size fractions with no obvious trends with particle size. Saharan spectra were distinct from North American spectra, which confirms the different compositions of the two sources. Marine spectra displayed a range of both North American and Saharan spectral characteristics likely reflecting that the marine samples are ultimately derived from these and potentially other sources. However, despite the notable variation in compositions, surface area is the predominant factor affecting total iron solubility. Aerosol iron solubilization through acidic surface reactions would be most effective in samples with the highest surface area, which likely results in the strong positive correlation with soluble iron concentration. 